\title{
Changes in surface topology of amorphous silicon oxide and mica after ion-milling
}

\author{
Manfred Radmacher, Monika Fritz-Stephan \& Hermann E. Gaub* \\ Physikdepartment der Technischen Universität München, 8046 Garching \\ - Present address: \\ Chemistry Department Stanford University Stanford Ca 94305
}

\begin{abstract}
The surface topology of amorphous silicon oxide and mica were investigated by Scanning Force Microscopy. The surface of thermally grown oxide films on silicon wafers appeared to be flat on the large scale but exhibited a wrinkled surface structure on the sub-nanometer scale. After ion milling in an argon plasma we found the surface roughness of the silicon oxide to be drastically increased on the $10 \mathrm{~nm}$ scale. Mica, however, responded differently to the ion milling. Here we found that after exposure to the argon plasma, small flakes of the layered mineral were left on the surface. The flakes were only loosely attached to the surface. Both on top of the flakes and on the undamaged surface, atomic resolution was achieved.
\end{abstract}

\section{INTRODUCTION}

Ion milling, also called plasma cleaning, is a widely used method for cleaning or sterilizing various surfaces. It is also one of the standard procedures in the preparation of electron microscope specimen. Here the plasma is used to deliberately alter the surface chemistry as well as the surface charge density of substrates like carbon films (Glaeser and others 1991). As these carbon films are manufactured by vapor deposition, they exhibit a surface roughness on the scale of typically several $\mathrm{nm}$. Investigations with STM have shown that these film show no significant change in the surface topology upon ion milling under various conditions, although their surface electrical properties as well as their water wetability is drastically changed (Glaeser and others 1991) (Guckenberger, private communication).

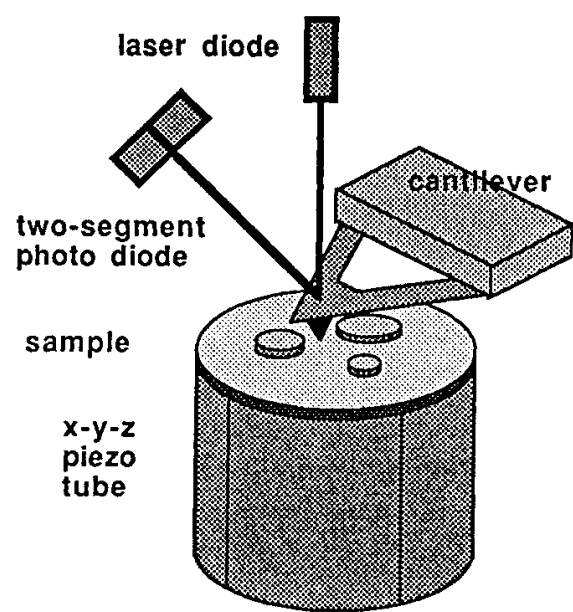

Fig. 1

Schematics of a Scanning Force Microscope

In order to investigate the influence of ion milling on substrates used for SFM (Hansma and others 1988)(Binnig and others 1986) which are in general flat nonconducting surfaces, we have investigated here the surface of the two most frequently used surfaces: mica and silicon oxide. These two samples also differ in that one is amorphous and the other one a highly ordered solid which might give some insight into the influence of crystallinity on the etching process. 


\section{MATERIALS AND METHODS}

The scanning force microscope measurements were performed with an home built instrument described in (Radmacher and others 1992). The schematics of such an instrument is depicted in Fig. 1. Commercially available silicon nitride cantilevers ( NanoProbes, Digital Instruments, Santa Barbara $\mathrm{Ca}$ ) with integrated tips were used. All images were taken in air except image $3 b$ which was taken under water. Forces were in the $10 \mathrm{nN}$ range. The silicon wafers, which were a kind gift of Wacker (Burghausen, FRG) were a $<100>$ p-type semiconductor with a thermally grown oxide layer of about $150 \mathrm{~nm}$ thickness. The wafers were cleaned in a bath sonicator in $2 \%$ Hellmanex (Hellma, Müllheim, FRG) for 30 minutes at $30^{\circ} \mathrm{C}$. Then they were rinsed 10 times with pure water (Milli $Q$ system, Molsheim, France) and again ultrasonicated in pure water for 30 minutes at room temperature. Mica was freshly cleaved before plasma cleaning by pealing off the top layer with scotch tape.

Plasma cleaning was performed with a commercially available plasma cleaner (Harrick PDC-3XG, New York) in pure argon atmosphere at about 1 mbar pressure. The exposure time to the plasma was on the order of some seconds.

\section{RESULTS AND DISCUSSION}

Because of its extreme flatness mica has developed to be one of the standard substrates for Scanning Force Microscopy. Under normal conditions, freshly cleaved mica appears completely structureless on the $\mu \mathrm{m}$ scale. On the $\mathrm{nm}$ scale the periodicity of the unit cell is usually resolved without problems. Mica is therefore regularly used for the calibration of the instrument. Fig 2 a shows such a freshly cleaved mica surface at high magnification. The hexagonal pattern has a repeat distance of $0.52 \mathrm{~nm}$. Details within the unit cell are not resolved here. After ion milling, however, the surface of the freshly cleaved mica was drastically altered. On the large scale the surface appeared to be covered with thin flakes (See Fig $2 b$ and $c$ ). The flakes exhibited thickness which appeared to be multiples of the single layer thickness of $2 \mathrm{~nm}$. The flakes seemed to be only loosely attached to the surface as some of the smaller flakes were laterally shifted on the surface upon scanning (See arrow Fig 2c). Not only on the large flat areas but also on top of the flakes, the structure of the mica lattice could be resolved. (See Fig. 2d). Probably due to the loose contact of the flake, the pattern appeared in the latter case to be slightly distorted and less well resolved. The structure on the large flat areas between the flakes was like the one shown in Fig 2a. From these findings we conclude that the surface of the layered mica crystal was cracked by the ion milling process which resulted in a break up of the upper layers into many flakes. This situation is similar to an anisotropic etching process. The smaller flakes were probably removed from the surface by the SFM tip while scanning, whereas the larger flakes, due to their stronger adherence, remained at the surface. It is astonishing however, that we could not image any cracks in the surface of the basis layer. The only crack that could be resolved after ion milling was on top of a flake. (See arrow Fig2c)

One of the reasons, that mica is such a prominent substrate for SFM is its extreme flatness together with its ease of use. It is on the other hand extremely difficult to chemically modify the mica surface in order to attach larger molecules to be imaged. Silicon oxide offers the advantage of a well developed surface chemistry (MaOz and Sagiv 1984) (Scouten 1987). Regular glass surfaces do unfortunately exhibit, in general, a surface roughness which is on the order of the size of the macromolekules like proteins (Radmacher and others 1991), which may result in images that are hard to interpret. Thermally grown silicon oxide on silicon wafers, however, have been show to be structureless on this scale, which makes them nicely suited substrates for SFM. Here we have studied the influence of argon ion milling on such surfaces. Fig. $3 b$ shows the surface of a silicon 100 crystal covered with a $150 \mathrm{~nm}$ thick layer of thermally grown amorphous oxide. It appears completely flat with a roughness of $<0.3 \mathrm{~nm}$ rms. A close-up view of this surface, however, exhibits the amorphous nature of the surface. (See Fig 3a) 
a)
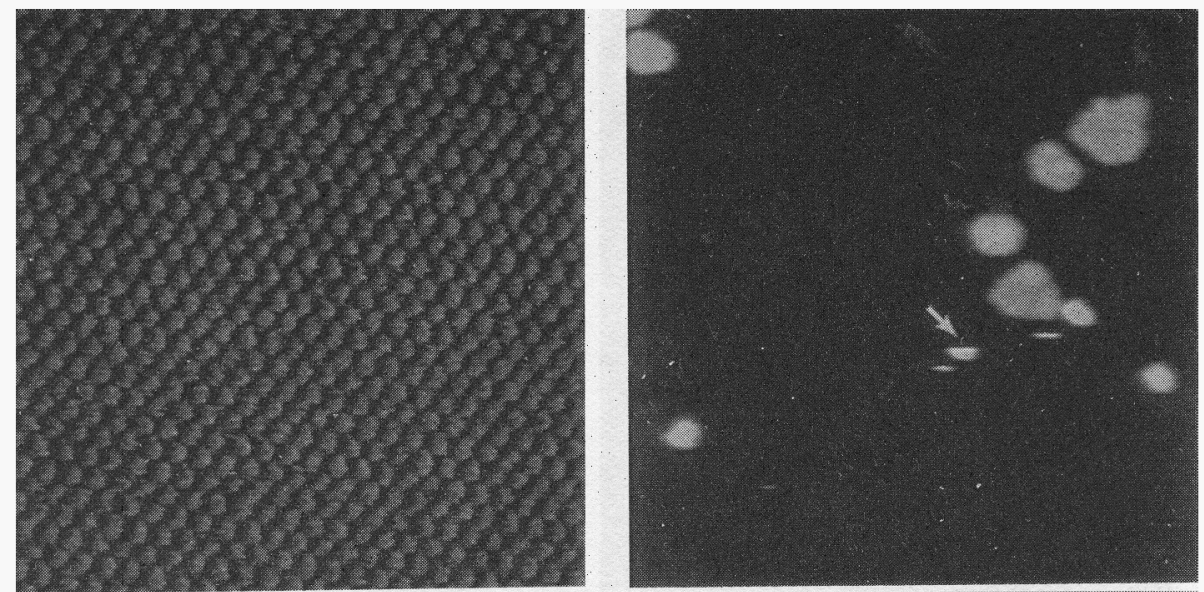

b)
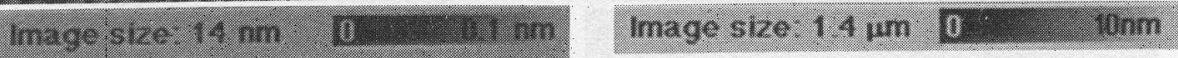

c)
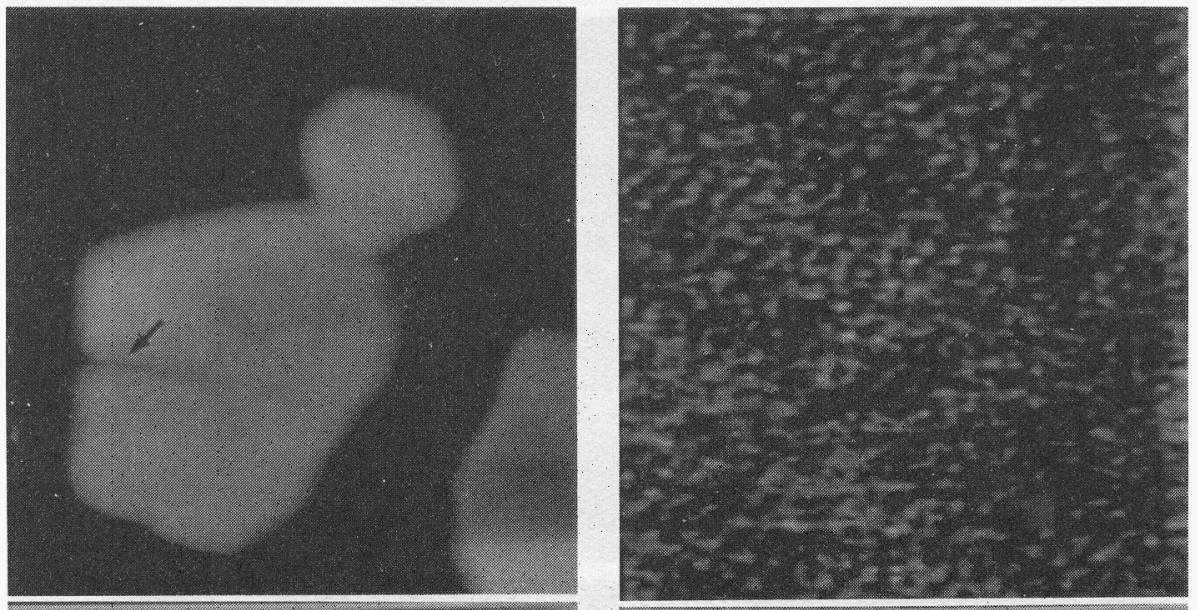

d)

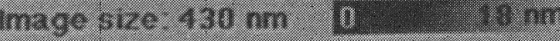

image size 14 nm $100001 \mathrm{~mm}$

Fig. 2

a) High magnification SFM image of ruby mica.

b) Same surface at lower magnification after argon ion milling. Note that the smaller flakes are moved during scanning (see arrow).

c) Close-up view of one of the flakes showing a crack (arrow).

d) High magnification of the surface on top of a larger flake.

The wrinkled surface structures have an average next neighbor distance of $0.4-0.5 \mathrm{~nm}$ and an average height of $0.05 \mathrm{~nm}$. We have good indications to believe that these surface structures represent the hydration shell of the surface rather than the silicon oxide surface itself (Radmacher and others 1992). The same surface was imaged after ion milling. The result is shown in Fig. 3c. The surface topology appears to have been drastically altered by the ion bombardment. It now exhibits holes with a depth of up to tens of $\mathrm{nm}$ and about the same lateral dimensions as well as caustic hills of several $\mathrm{nm}$ in height. Ellipsometry measurements of the thickness of comparable wafers give no indication for a significant decrease of the thickness of the oxide layer (H.E.G. unpublished data). Even after ion milling of several minutes the thickness stays constant to within several nanometers. This means that either the silicon oxide is removed from the surface at an very slow rate, or that the surface deformation is dominated by a scrambling of the surface rather than an etching. 
a)

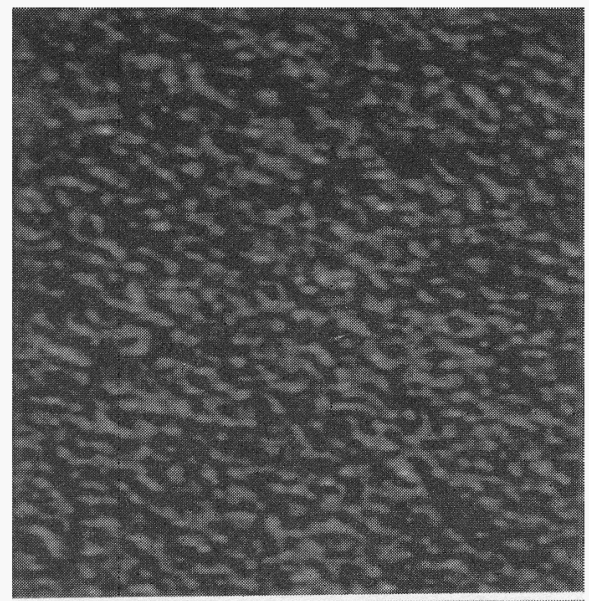

Image sizo: $14 \mathrm{~nm}$.0
Fig.3

a) High magnification of the surface of a silicon wafer with a thermally grown silicon oxide.

b) Low magnification image of the same surface.

c) Same surface after high pressure argon ion milling.
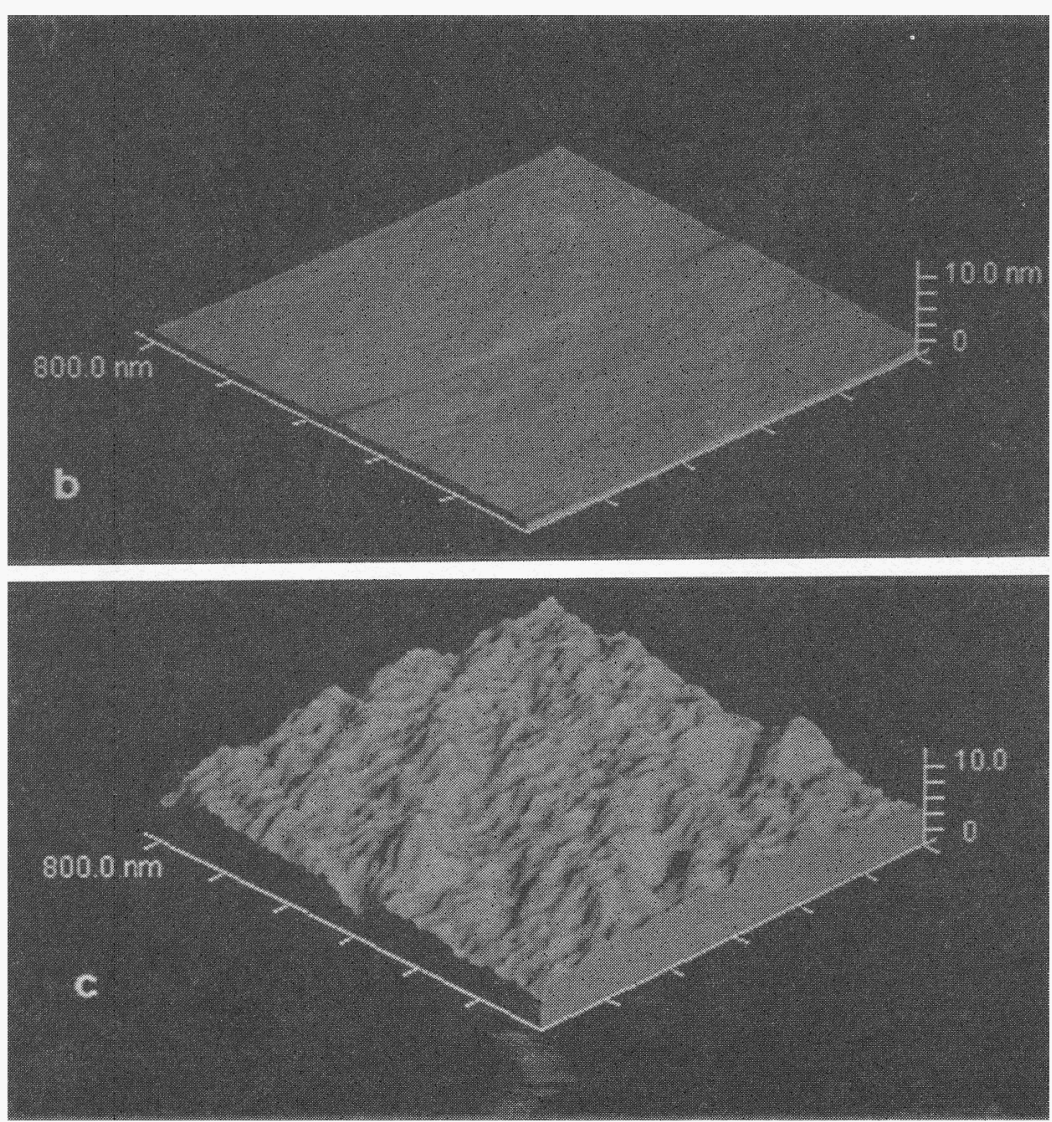

\section{CONCLUDING REMARKS}

Plasma cleaning of mica and silicon oxide surfaces may obviously result in a massive change of the surface topology. This finding is in contrast to the results found by Ducker et al (Ducker and others 1991) who worked at much lower pressures of the gas. Under these conditions they found virtually no increase in surface roughness. Our study shows that at higher pressures in the case of mica, the first layers are more or less completely removed so that after the plasma cleaning the surface is essentially flat and covered with 
crystalline mica flakes. In the case of the silicon oxide the surface is roughened considerably. Our results show clearly that this kind of surface treatment is not useful for sample preparation in SFM but it also shows very clearly that this procedure when applied to the AFM tip increases the surface roughness of the tip, which in certain cases gives rise to higher resolution.

\section{Acknowledgements}

This work was supported by the Deutsche Forschungsgemeinschaft. We thank Dominic Benvegnu for correcting this manuscript.

\section{REFERENCES}

Binnig, G, C.F. Quate, and Ch Gerber. "Atomic Force Microscope." Phys, Rev, Lett 56 (1986): $930-$

Ducker, W A., Tim J. Senden, and Richard M. Pashley. "Direct measurement of colloidal forces using an atomic force microscope." Nature 353 (19 September 1991): 239-241.

Glaeser, R. M. , A. Zilker, M. Radmacher, H.E. Gaub, T. Hartmann, and W. Baumeister. "Interfacial Energies and Surface Tension Forces Involved in the Preparation of Thin, Flat Crystals of Biological Macromolecules for High Resolution Electron Microscopy." $\mathbb{L}$. of Microscopy 161 (1991): 21-45.

Hansma, P.K., V.B. Elings, O. Marti, and C.E. Bracker. "Scanning Tunneling Microscopty and Atomic Force Microscopy; Applications to Biology and Technology." Science 242 (1988): 209.

Maoz, R. and J. Sagiv. "On the formation and structure of self-assembling monolayers." $\underline{\mathrm{J}}$ Colloid Interface Sci 100 (2 1984): 465-496.

Radmacher, M, K Eberle, and H.E. Gaub. "An AFM with Integrated Micro Fluorescence Optics - Design and Performance." Ultramicroscopy (1992):

Radmacher, M. , B.M. Goettgens, R.W. Tillmann, H.G. Hansama, P.K. Hansma, and H.E. Gaub. "Morphology of Polymerized Membranes on an Amorphous Substrate at Molecular Resolution by AFM." In Scanned Probe Microscopies, ed. K.Wichramasinghe \& F.A. McDonald. 1991.

Radmacher, M, R Zimmermann, and H.E Gaub. "Why can the Atomic Force Microscope image individual Lipids." In Amphiphilic membranes, ed. Richter and R. Lipowsky. Springer, 1992.

Scouten, W. H. "A survey of Enzyme Coupling Techniques." In Methods in Enzymology, ed. Klaos Mosbach. 30-64. 135. Academic Press, 1987. 